\title{
Role of Fine Needle Aspiration Versus Non-Aspiration Cytology In Diagnosis of Thyroid Lesions
}

\author{
Bhavneet Kour $^{1}$, Kuldeep Singh ${ }^{2}$, Pankaj Singh ${ }^{3}$ \\ ${ }^{I}$ (Department of Pathology, Government Medical College, Jammu, $J \& K$, India) \\ ${ }_{2}^{2}$ (Department of Pathology, Government Medical College, Jammu, J\&K, India) \\ ${ }_{3}^{3}$ (Department of Medicine, Government Medical College, Jammu, J\&K, India)
}

\begin{abstract}
Background: Fine needle aspiration cytology $(F N A C)$ is the first choice for evaluation of thyroid enlargement. Diagnosis is based on the identification of predominant cell pattern, cell morphology and background details. Fine needle non-aspiration cytology (FNNAC) avoids aspiration, uses capillary action of the fine needle. It is more patient friendly with less pain and better perception of lesion.

Aim of the study: To evaluate the results offine Needle Aspiration Cytology (FNAC) and Fine Needle Non Aspiration Cytology (FNNAC) in diagnosis of thyroid lesions.

Materials and Methods: One-year observational, prospective study was conducted on patients with palpable thyroid lesions in the Department of Pathology, Government Medical College, Jammu. Patients were investigated with a thyroid function test and a needle biopsy. Both FNAC and FNNAC techniques were performed simultaneously at the same site by the same investigator.

Points were allocated to each specimen according to Mair et al. scoring system and categorized as follows: unsuitable for cytodiagnosis (0-2 points), diagnostically adequate (3-6 points) and diagnostically superior (7-10 points).

Results: A total of 140 patients with palpable thyroid lesions were subjected to needle biopsies. Females dominated the study with female to male ratio of 5.36:1. Non-neoplastic lesions were observed in $89.29 \%$ and neoplastic in $10.71 \%$ cases. . The cumulative score was found significantly more in case of FNNAC than FNAC (946 vs 784; p <0.0001). For diagnostically superior category, results of both FNAC and FNNAC were comparable.

Conclusion: FNAC smears produced adequate results in more cases. FNNAC was more diagnostically superior. It causes less damage to tissue, less artefacts and allows better perception of lesion. FNNAC is a good technique that should be used alone or in combination with FNAC for better diagnostic yield.
\end{abstract}

Keywords: Thyroid lesions, Fine needle aspiration, Fine needle non-aspiration, Cytology

\section{Introduction}

Thyroid enlargement is a common occurrence in most regions of the world. India has the world's biggest goiter belt in the sub-Himalayan region including the state of Jammu and Kashmir. In these iodinedeficient areas, the incidence of goiter among thyroid nodules is much higher $(\sim 40 \%)$. Thyroid diseases are not uncommon in our environment of study and being a referral hospital in Jammu Division, patients are referred for further management. Thyroid enlargement, whether diffuse or in the form of a nodule, leads to a battery of investigations, mainly to rule out the possibility of a neoplasm or thyroiditis. Fine-needle aspiration (FNA) cytology is the cornerstone of thyroid nodule evaluation. It can be safely performed on an outpatient basis. FNAC of the thyroid has become increasingly popular over last four decades (1). For thyroid lesions, FNAC is first investigation of choice as the thyroid enlargement either diffuse or nodular can occur in any thyroid lesion like colloid goitre or thyroiditis or neoplasm (2). Accurate diagnosis of various thyroid lesions by FNAC is based on the identification of predominant cell pattern, cell morphology and background details (3). There is another technique, however, fine needle capillary (FNC) sampling/ fine needle non-aspiration cytology (FNNAC) which avoids aspiration but still permits cytologic review of the masses. This technique depends solely on capillary action of the fine needle, therefore is less painful, less traumatic and thus much more patient friendly (4).Several studies have reported conflicting results between FNAC and FNNAC $(5,6,7)$. Some studies have reported that FNNAC produces better specimens for cytological diagnosis (7).

Both, FNAC and FNNAC methods have advantages and disadvantages but, as yet, there is no agreement on which method produces better specimens for cytological diagnosis. The present study was carried out to evaluate theresults of FNAC and FNNAC in cytological diagnosis of thyroid lesions. 


\section{Materials and Methods}

The present observational prospective one-year study was conducted on patients with a thyroid swelling/nodule in the Government Medical College Hospital, Jammu. All the cases of palpable thyroid lesionsirrespective of consistency, size of lesions, age and sex of the patients referred to the Department of Pathology by various clinical departments including outpatient and indoor patients of Government Medical College and Associated Hospitals, as well as patients referred from other hospitals were subjected to both FNAC and FNNAC techniques.Study was conducted after obtaining necessary approval from the Institutional Ethics Committee. Exclusion criteria included cases of non-palpable thyroid lesions requiring image guided FNA and patients not willing to participate in the study.

After detailed clinical examination along with ensuring to note any doubtful sites on the thyroid gland, the patient was investigated with a thyroid function test and a needle biopsy. The patient was subjected to needle biopsies after obtaining informed written consent. Both FNAC and FNNAC techniques were performed simultaneously at the same site by the same investigator. An average of 4 slides was obtained by both the techniques.

\subsection{Method}

Fine Needle Aspiration Cytology:

1. With the patient in comfortable position, swelling was grasped with two fingers of one hand and prepared by applying an antiseptic solution.

2. The aspiration was performed with a fine needle of $21-23$ gauge attached to $20 \mathrm{ml}$ disposable syringe and fitted to Cameco pistol.

3. After the needle was well advanced in the swelling, adequate negative pressure wias exerted varying with the density of the specimen to be aspirated.

4. With the suction held steady, the needle was moved back and forth within the lump using short quick strokes and released the pistol of the syringe before removing the needle.

5. Needle was withdrawn gently and immediate pressure was applied to the puncture site with a sterile gauze pad.

6. Syringe was filled with air after removing the needle and sample was expressed onto the slides after reattaching the needle.

7. Some of the smears were fixed in alcohol and some air-dried.

\section{Fine Needle Non-Aspiration Cytology:}

Non-aspiration needle sampling was obtained by inserting the needle held between thumb and forefinger of the one hand into the swelling and moving the needle rapidly in and out within the mass as with conventional fine needle aspiration method but without attachment to a syringe or a holder. Upon withdrawal of the needle, a syringe filled with air was then attached to the needle to enable expression of needle contents onto the glass slide. Some of the smears were fixed in alcohol and some air-dried. Staining was conducted by May Grunwald Giemsa (MGG) (8) and Papanicolaou (PAP) staining (9) methods.

\subsection{Specimen quality analysis} follows:

On these slides, using point scoring system of Mair et al. (10), all the specimens were classified as

$\begin{array}{ll}\text { i. } & \text { Criterion } \\ & \text { Background } \\ \text { Blood or clot }\end{array}$

ii. Amount of cellular materia

iii. Degree of cellular degeneration

\section{Qualitative description}

Large amount:

Great compromise in diagnosis

Moderate amount:

Minimal:

Diagnosis possible

Diagnosis easy specimen of

text book quality

Minimal to absent:

Diagnosis not possible

Abundant:

Sufficient for diagnosis 1

Marked:

Diagnosis simple

Moderate:

\section{Point scores}

0

2

0 
Minimal:

Good preservation diagnosis

easy

Degree of
cellular
trauma

5. Retention of appropriate architecture

Marked:

Moderate:

Diagnosis not possible

Diagnosis possible

Minimal:

Diagnosis obvious

Minimal to absent:

Non diagnostic

Moderate:

Some preservation e.g., follicles,

papillae, acini, flat sheets,

syncitia or single cell pattern

Excellent architecture display

closely reflecting histology:

Diagnosis obvious

On the basis of these five criteria tabulated, a cumulative score between 0 to 10 points was allocated to each fine needle specimen which was then categorized according to one of the three categories

- Unsuitable for cytodiagnosis

- Diagnostically adequate

$0-2$ total points

- Diagnostically superior

$$
\begin{aligned}
& 3-6 \text { total points } \\
& 7-10 \text { total points }
\end{aligned}
$$

a. Unsuitable specimens: Consist mainly of red blood cells or absent cellularity making them inadequate for cytodiagnosis.

b. Diagnostically adequate: Possible to render an opinion on nature of lesion sampled but the cellular material present can be sub-optimal due to poor cellularity, sample dilution, degenerative changes, or specimen entrapment in blood clots.

c. Diagnostically superior: Cell aggregates are prominent, well preserved, and unobscured by background blood and cellular morphology is well displayed. Data was expressed as mean \pm standard deviation. The qualitative data was compared using chi-square test. For comparison of means, unpaired ' $t$ '-test was performed. A p-value less than 0.05 was considered as level of significance.

\section{Results}

A total of 140 patients with palpable thyroid lesions were subjected to needle biopsies using both FNAC and FNNAC techniques. The study observed female $(118 ; 84.29 \%)$ dominted male $(22 ; 15.71 \%)$ patients, with female to male ratio of 5.36:1. Mean age of the patients was 38.17 years (range 2 to 90 years), median age being 37.5 years. Most of the patients were in their $3^{\text {rd }}(42 ; 30 \%)$ decade of life, followed by $2^{\text {nd }}(27$; $19.29 \%), 4^{\text {th }}(20 ; 14.29 \%)$ and $5^{\text {th }}(16 ; 11.43 \%)$ decade of life. A total of $125(89.29 \%)$ thyroid lesions were non-neoplastic, while $15(10.71 \%)$ thyroid lesions were neoplastic in nature.

Frequency of thyroid lesions diagnosed is given in Table 1.

Table 1: Frequency of thyroid lesions

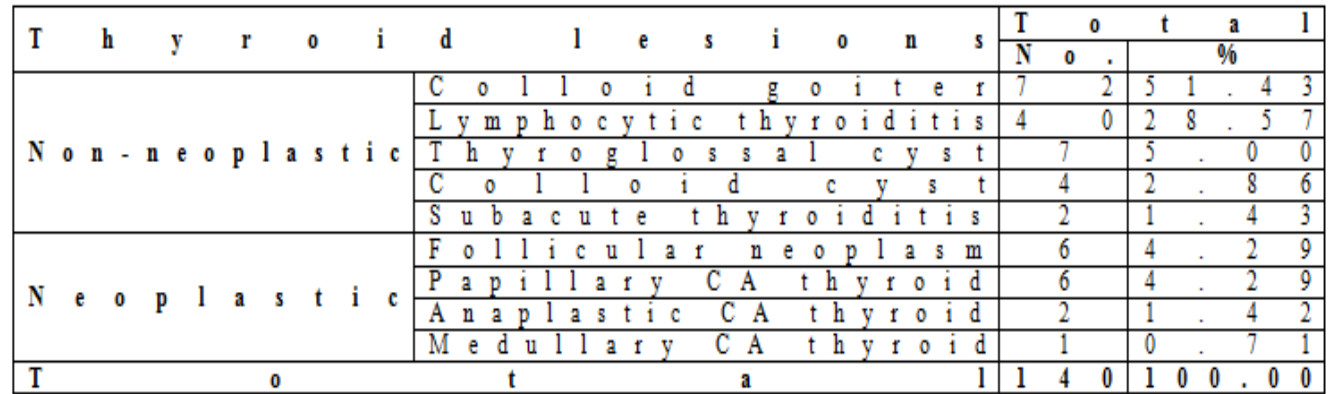


Comparison of FNAC and FNNAC of thyroid lesions on the basis of modified scoring system is given in Table 2, while comparison of diagnostic quality between FNAC and FNNAC of thyroid lesions is given in Table 3.

Table 2: Comparison of FNAC and FNNAC of thyroid lesions on the basis of modified scoring system

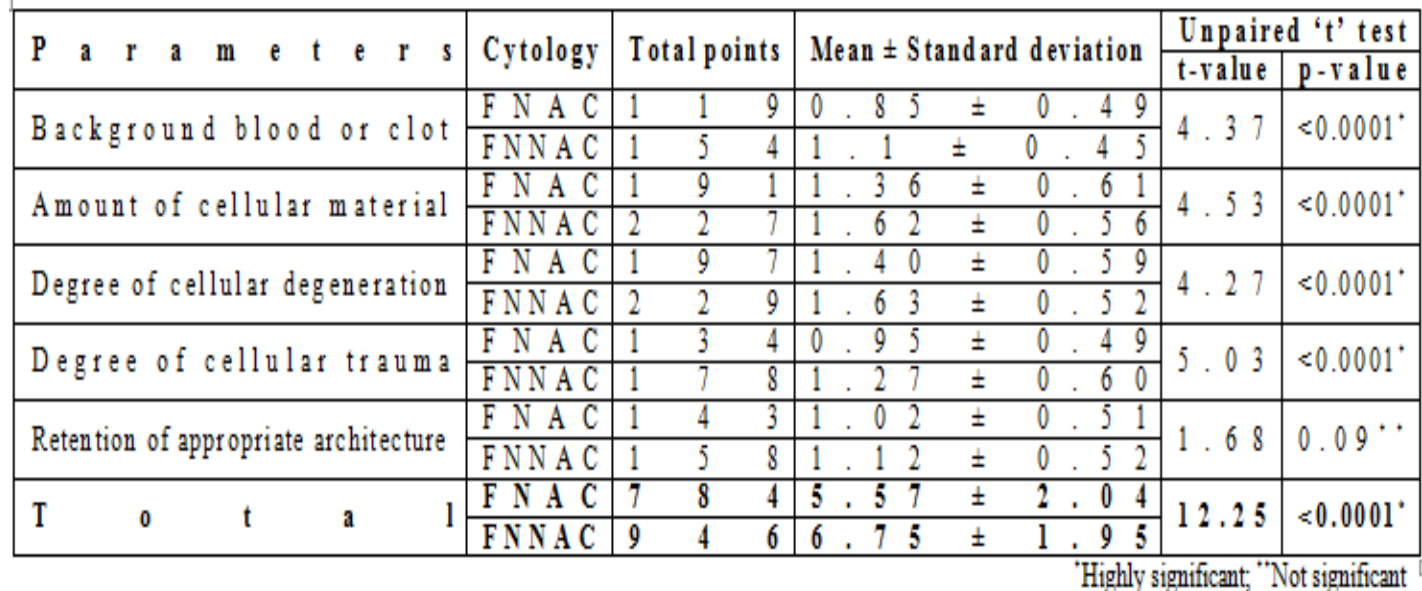

Table 3: Comparison of diagnostic quality between FNAC and FNNAC of thyroid lesions

\begin{tabular}{|c|c|c|c|c|}
\hline & $\mathrm{F}$ & $\mathrm{A}$ & $\mathrm{F} \quad \mathrm{N}$ & $\mathrm{A}$ \\
\hline 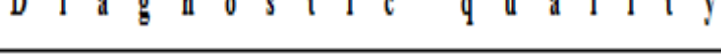 & $\mathrm{N} 0$ & $\%$ & N 0 & $\%$ \\
\hline cytodiagnosis & 1 & 10 & 6 & .2 \\
\hline Diagnostically adequate & 6 & 54. & & 30.7 \\
\hline Diagnostically & 49 & 35.00 & $9 \quad 1$ & 65.0 \\
\hline $\begin{array}{lll}\mathrm{T} & 0 & \mathrm{t} \\
\end{array}$ & 140 & 100.00 & 140 & 100.0 \\
\hline
\end{tabular}

Correlation of diagnostic quality between FNAC and FNNAC is shown in Table 4.

Table 4: Correlation of diagnostic quality between FNAC and FNNAC of thyroid lesions

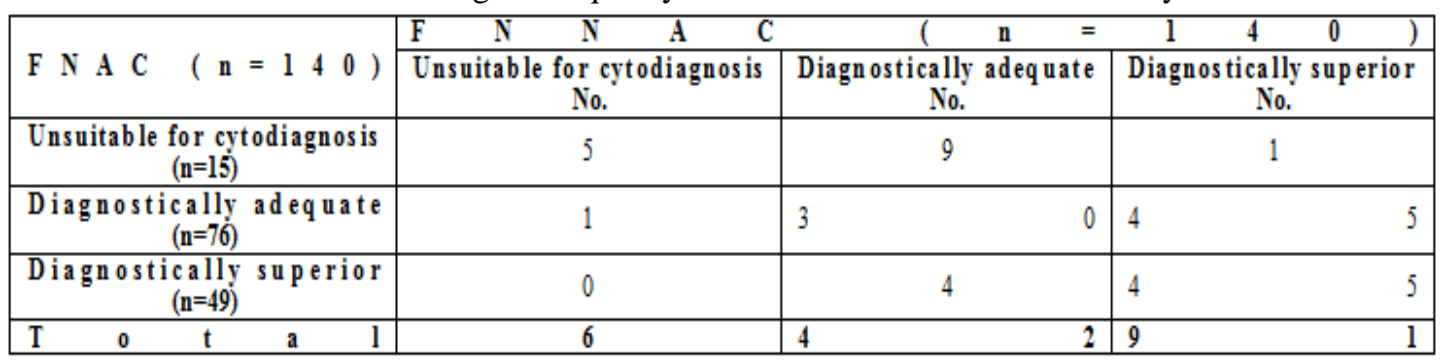

\section{Discussion}

Thyroid disorders vary in terms of their presentation and characteristics. Thyroid swellings/nodules demand a sequence of investigations to rule out the possibility of a neoplasm or thyroiditis (11).FNAC is an important tool for the cytological assessment of patients with superficial as well as deep seated lesions. But this technique is frequently complicated by aspiration of significant quantities of blood particularly in vascular organs or haemorrhagic tumours which compromise cellular preservation and interpretation. In an attempt to overcome this problem a non-aspiration technique, called FNNAC was pioneered in France. FNNAC technique employs insertion of fine needle into lesions without attachment of a syringe. It relies on the property of capillary tension in narrow channel. In the present study, most of the patients were in their third decade of life (30\%). Mean age of the patients was 38.17 years, median age being 37.5 years. Female patients $(84.29 \%$ dominated the study with female to male ratio of 5.36:1 i.e., females were five times more vulnerable of developing thyroid lesions as compared to males. Tayde et al. (1) and Jain et al. (12) also reported similar findings. A total of $125(89.29 \%)$ of thyroid lesions were non-neoplastic, comprising of $72(51.43 \%)$ colloid goiter, 40 (28.57\%) lymphocytic thyroiditis, 7 (5\%) thyroglossal cyst, $4(2.86 \%)$ colloid cyst and $2(1.43 \%)$ subacute thyroiditis cases. Neoplastic lesions were diagnosed in $15(10.71 \%)$ cases, comprising of follicular neoplasm and papillary carcinoma thyroid in 6 (4.29\%) cases each, anaplastic carcinoma thyroid in $2(1.42 \%)$ 
cases and medullary carcinoma thyroid in $1(0.71 \%)$ case. Similar to the present study, Mahajan and Sharma (13) reported that non-neoplastic lesions comprised $80 \%$ of the total cases $(n=50)$ comprising mainly of colloid goiter (19 cases), benign thyroid hyperplasia (6 cases), lymphocytic thyroiditis (13 cases) and granulomatous (2 cases). Out of the 10 neoplasms (20\%), 6 were follicular neoplasms, 2 cases of medullary and 2 of papillary carcinoma. The present study assessed smears of FNAC and FNNAC using modified scoring system (10). Both FNAC and FNNAC yielded good material for diagnosis of thyroid lesions. The cumulative score was found significantly more in case of FNNAC than FNAC (946 vs 784; p<0.0001). FNNAC was extremely useful in non-neoplastic and neoplastic thyroid lesions as the needle held directly in the fingers acted as sensor, providing tactile information which is not yielded when syringes and holders are employed. Moreover, the method caused minimal pain due to reduced trauma to the secondary tissues.It was also found in the present study that contamination with blood was more in malignancies in FNAC samples compared to FNNAC samples because of vascular nature of tumour tissue and malignant cells being fragile were more prone to degeneration and trauma of suction. Moreover, in the present study, the cellular yield in the thyroid lesions was higher for FNNAC as compared to FNAC.

Romitelli et al. (7) observed a statistically significant difference in the total score in favour of nonaspiration as compared with aspiration technique, with FNNAC producing a better quality of specimen. Tauro et al. (11)also observed that on the parameter of blood clots, FNNAC produced least likelihood of haemorrhage, thereby giving a clear picture to the cytopathologist. Both the studies reported results which are similar to the present study.Kaur et al. (14) also found better average score per case by non-aspiration technique for cellular trauma, degenerative changes and less likelihood of obscuring by blood, which is similar to the present study.

Like present study, Pandey et al. (15) also reported that results for background blood and cellular material were highly significant in favour of FNNAC as compared to FNAC. FNNAC was found to cause lesser degree of contamination because specimen was obtained by a spontaneous capillary action rather than suction due to negative pressure. The authors also found that FNNAC performed far better than FNAC for malignant lesions i.e., yielded adequate material with less contamination of blood, which is similar to findings of the present study. They also observed that mean scores for degree of cellular degeneration and trauma were more for FNNAC as compared to FNAC, which is in agreement with our study.Findings similar with our study were also reported by Mahajan and Sharma (13), Rizvi et al. (16), and Maurya et al. (17).

Ramachandra et al. (18)observed that FNNAC yielded more diagnostically better specimens as compared with FNAC and the number of unsuitable smears was also greater in FNAC, which is similar to our study.In the study of Chowhan et al. (19),non-aspiration technique yielded less diagnostically adequate but more diagnostically superior smears when compared with aspiration technique. These findings are in consonance with our study. When diagnostic results of FNAC were correlated with those of FNNAC, the present study observed that out of 15 thyroid lesions found unsuitable for cytodiagnosis by FNAC, FNNAC showed 5 as unsuitable, 9 as adequate and 1 thyroid lesion as superior. Similarly, out of 76 thyroid lesions found adequate by FNAC, FNNAC showed 1 as unsuitable, 30 as adequate and 45 as superior. However, for diagnostically superior, results of both FNAC and FNNAC were comparable. While FNAC found 49 thyroid lesions as superior, FNNAC results were in agreement for 45 lesions, rest 4 were shown as adequate. Both FNAC and FNNAC have their own advantages and disadvantages. FNNAC offers distinct advantage of diagnostically better quality smears, while FNAC assures diagnostically adequate material quantitatively. The technical ease of capillary sampling may prompt adoption of FNNAC sampling of organs with higher vascularity.

\section{Conclusion}

FNAC smears produced adequate results in more cases and also simple benign lesions (e.g. colloid cyst) can be drained by aspiration technique for therapeutic purpose. In highly cellular lesions in which abundant material was obtained, FNNAC was more diagnostically superior. Non-aspiration technique is simple, easy to perform with better patient compliance, especially in children. It causes less damage to tissue, less artefacts and allows better perception of lesion. FNNAC is a good technique that should be used alone or in combination with FNAC for better diagnostic yield.

\section{References}

[1]. Tayde A, Kulkarni V, Hiwale K. Role of cytology in thyroid lesions: A comparative study of fine needle aspiration and non aspiration techniques. J Cont Med A Dent 2014; 2(2): 39-44.

[2]. Amrikachi M, Ramzy I, Rubenfeld S, Wheeler TM. Accuracy of fine needle in clinical practise. Thyroid Arch Pathol Lab Med 2001; 125: 484-8.

[3]. Bommanahalli BP, Bhat RV, Rupnarayan R. A cell pattern approach to interpretation of the fine needle aspiration cytology of thyroid lesions. A cyto-histomorphological study. J Cytol 2010; 27: 127-32.

[4]. Raghuveer CV, Leekha I, Pai MR, Adhikari P. Fine needle aspiration cytology versus fine needle sampling without aspiration: A prospective study of 200 cases. Indian J Med Sci 2002; 56: 431-9.

[5]. Kamal MM, Arjune DG, Kulkarni HR. Comparative study of fine needle aspiration and fine needle capillary sampling of thyroid lesions. Acta Cytol 2002; 46: 30-4. 
[6]. Haddadi-Nezhad S, Larijani B, Tavangar SM, Nouraei SM. Comparison of fine - needle - nonaspiration with fine - needle aspiration technique in the cytologic studies of thyroid nodules. Endocr Pathol 2003; 14: 369-73.

[7]. Romitelli F, Di Stasio E, Santoro C, et al. A Comparative Study of Fine Needle Aspiration and Fine Non Aspiration Biopsy on suspected thyroid modules. Endocr Pathol 2009; 20: 108-13.

[8]. Bales CE. Laboratory techniques. In: Koss LG, Melamed MR (editors), Koss's Diagnostic Cytology and Its Histopathological Bases, $5^{\text {th }}$ edition. Philadelphia: Lippincott Williams and Wilkins, 2006; vol. 2: p. 1616.

[9]. Morse A. Diagnostic cytopathology specimen collection and preparation. In: Bancroft JD, Gamble M (editors), Theory and Practice of Histological Techniques, $5^{\text {th }}$ edition. Churchill Livingstone, 2002: pp. 631-2.

[10]. Mair S, Dunbar F, Becker PJ, Du Plessis W. Fine needle cytology - Is aspiration suction necessary? A study of 100 masses in various sites. Acta Cytol 1989; 33: 809-13.

[11]. Tauro LF, Lobo GJ, Fernandes H, George C, Aithala PS, Shenoy D, et al. A comparative study on fine needle aspiration cytology versus fine needle capillary cytology in thyroid nodules. Oman Med J 2012; 27(2): 151-56.

[12]. Jain S, Nayak R, Totade S, Shukla N. Clinico-pathological correlation of thyroid swellings. Int J Med Res Rev 2014; 2(6): 553-60.

[13]. Mahajan P, Sharma PR. Fine-needle aspiration versus non-aspiration technique of cytodiagnosis in thyroid lesions. JK Science 2010; 12(3): 120-22.

[14]. Kaur S, Garg U, Kaur S, Singh K, Kamra H, Verma S. Comparison of aspiration vs non-aspiration techniques in fine-needle cytology of thyroid lesions. IOSR-JDMS 2014; 13(5): 97-101.

[15]. Pandey P, Dixit A, Aggarwal R, Mahajan NC. Fine needle aspiration cytology versus fine needle capillary sampling in cytologic al diagnosis of thyroid lesions. Iranian J Pathol 2015; 10(1): 47-53.

[16]. Rizvi SAA, Hasain M, Khan, et al. A comparative study of the needle aspiration cytology versus non-aspiration technique in thyroid lesions. Surgeon 2005; 3(4): 273-76.

[17]. Maurya AK, Mehta A, Mani NS, Nijhawan VS, Batra R. Comparison of aspiration vs non-aspiration techniques in fine-needle cytology of thyroid lesions. J Cytol 2010; 27(2): 51-4.

[18]. Ramachandra L, Kudva R, Anand Rao BH, Agrawal S. A comparative study of fine needle aspiration cytology (FNAC) and fine needle non-aspiration cytology (FNNAC) technique in lesions of thyroid gland. Indian J Surg 2011; 73(4): 287-90.

[19]. Chowhan AK, Sreedhar Babu KV, Sachan A, Patnayak R, Radhika K, Phaneendra BV, et al. Should we apply suction during fine needle cytology of thyroid lesions? A prospective study of 200 cases. J Clin Diagn Res 2014; 8(10): FC19-22.

Bhavneet Kour. "Role of Fine Needle Aspiration Versus Non-Aspiration Cytology In Diagnosis
of Thyroid Lesions." IOSR Journal of Dental and Medical Sciences (IOSR-JDMS) 16.7 (2017):
99-104. 